\title{
AVALIAÇÃO DA QUALIDADE DE VIDA DE ESTUDANTES DE NUTRIÇÃO
}

\author{
EVALUATION OF THE QUALITY OF LIFE OF NUTRITION STUDENTS
}

EVALUACIÓN DE LA CALIDAD DE VIDA DE LOS ESTUDIANTES DE NUTRICIÓN

\author{
Solange Baraldi ${ }^{1}$ \\ Luciana Neves da Silva Bampi ${ }^{2}$ \\ Mariana Fernandes Pereira ${ }^{3}$ \\ Dirce Bellezi Guilhem ${ }^{4}$ \\ Aline Brandão Mariath ${ }^{5}$ \\ Ana Carolina Oliveira Campos ${ }^{6}$
}

Resumo Este estudo objetivou avaliar a qualidade de vida dos estudantes de Nutrição, de modo a subsidiar reflexões no processo de formação em saúde. Tratou-se de estudo observacional de corte transversal, realizado com quarenta estudantes distribuídos em todos os semestres do curso no período de agosto de 2010 a agosto de 2011. A coleta de dados foi realizada por meio de questionário específico - aspectos sociodemográficos - e do instrumento World Health Organization Quality of Life Instrument Bref. Foram realizadas análises estatísticas descritivas de frequência, tendência central e dispersão e análise inferencial de comparação entre os domínios. Estes apresentaram os seguintes escores: meio ambiente $(68,17)$, relações sociais $(66,67)$, físico $(66,07)$ e psicológico $(63,82)$. As facetas sono, energia para o dia a dia, capacidade de concentração, oportunidades de atividades de lazer, recursos financeiros, ambiente físico e sentimentos negativos influenciaram negativamente a qualidade de vida dos entrevistados e interferiram no desempenho acadêmico. Esses achados corroboram outros estudos sobre graduandos, revelando que a qualidade de vida desses jovens demanda aprimoramento das estruturas físicas e ambientais, do apoio pedagógico, social e psicológico num contexto de inclusão social e multidiversidade cultural cada vez mais presente nas universidades públicas brasileiras.

Palavras-chave qualidade de vida; percepção; estudantes; graduação; Nutrição.
Abstract This study aimed to evaluate the quality of life of Nutrition students in order to subsidize reflections on the process of training in health. This was a cross-sectional observational study conducted from August 2010 to August 2011 among forty students attending all course semesters. Data was collected using a specific questionnaire - sociodemographic characteristics - and the World Health Organization Quality of Life Instrument-Bref. Descriptive frequency, central tendency, and dispersion statistical analyses were performed, as was the comparison inferential analysis among the domains. The scores were: environment (68.17), social (66.67), physical (66.07) and psychological (63.82) relations. The sleep, energy for daily activities, concentration capacity, opportunities for leisure activities, financial resources, physical environment and negative feeling facets affected the respondents' quality of life adversely and interfered with their academic performance. These findings corroborate other studies involving undergraduates, revealing that the quality of life of these young people require improving physical and environmental structures and pedagogical, social, and psychological support in a context of social inclusion and of cultural multidiversity that is increasingly present in Brazilian public universities.

Keywords quality of life; perception; students; undergraduate courses; Nutrition. 


\section{Introdução}

De acordo com a Organização Mundial da Saúde (OMS), cuja missão principal é desenvolver ao máximo o nível de saúde de todos os povos, a saúde é definida como um "estado de completo bem-estar físico, mental e social, e não consiste apenas na ausência de doença ou de enfermidade" (Organização Mundial da Saúde, 1946). Embora seja um conceito amplo, nem sempre possível de mensurar em nível individual ou coletivo, ainda não foi substituído por um que seja internacionalmente aceito (Almeida Filho, 2000).

No entanto, a ausência de um conceito formalmente ampliado não tem limitado a compreensão ou mesmo a aplicação da noção de saúde por estudiosos ou pelos diversos sistemas de saúde no mundo. Dessa forma, é possível reconhecer a própria qualidade de vida como uma expressão da compreensão da saúde como uma categoria permeada por outros indicadores, que não apenas os biológicos ou os de morbimortalidade das populações. No Brasil, tal noção é demarcada na Constituição Federal Brasileira (Brasil, 1988), que conceitua saúde como um direito de todos e um dever do Estado, com acesso universal e igualitário.

Considerando essa complexidade, um grupo de estudiosos da OMS, denominado The World Health Organization Quality of Life Group, propôs um conceito subjetivo e multidimensional para qualidade de vida, que inclui elementos de avaliação positivos e negativos e que ficou assim definido: “Qualidade de vida é a percepção do indivíduo de sua posição na vida, no contexto da cultura e sistemas de valores nos quais vive, e em relação aos seus objetivos, expectativas, padrões e preocupações" (Organização Mundial da Saúde, 1998). Trata-se, portanto, de uma concepção ampla e complexa, que engloba a saúde física, o estado psicológico, o nível de independência, as relações sociais, as crenças pessoais e a relação com as características do meio ambiente, permitindo uma aproximação do conceito de saúde de modo transversal. Nesse sentido, a qualidade de vida reflete a percepção que os indivíduos têm de que suas necessidades estão sendo satisfeitas ou, contrariamente, de que lhes estão sendo negadas oportunidades de alcançar a felicidade e a autorrealização, com independência de seu estado de saúde físico ou das condições sociais e econômicas (Organização Mundial da Saúde, 1998).

A despeito de serem conceitos abrangentes - saúde e qualidade de vida-, eles geram debates e controvérsias em suas expressões e valores fundamentais. Algumas das inquietações sobre o estado de bem-estar ou de qualidade de vida perpassam as vivências do meio acadêmico na área de saúde. Parte dessas preocupações ocorre, especialmente, pelo fato de que o objeto central de trabalho desse futuro profissional estará relacionado ao atendimento das necessidades de prevenção, promoção ou recuperação do estado de 
saúde do ser humano. Tal preocupação é expressa claramente nas Diretrizes Curriculares Nacionais dos Cursos de Graduação em Enfermagem, Medicina e Nutrição (Brasil, 2001), que exigem que o universitário tenha uma formação profissional competente, humanista, ética, crítica e reflexiva.

A formação em saúde, por longo tempo, priorizou em suas grades curriculares um conhecimento predominantemente tecnicista e pouco integrado ao contexto do Sistema Único de Saúde (Ceccim e Feuerwerker, 2004), mas esta visão tem sido aprimorada ao longo de reflexões, críticas e focalização das políticas públicas. Assim, a publicação das Diretrizes Curriculares Nacionais dos Cursos de Graduação em Enfermagem, Medicina e Nutrição (Brasil, 2001) avança no sentido de exigir uma abordagem humanista e contextual, visando superar os desafios para o aprimoramento da qualificação profissional em saúde. Embora tenha se tornado um curso superior, de certa forma, recentemente, a Nutrição também passou por essa trajetória e almeja um perfil profissional imbuído de formação generalista, crítico e reflexivo (Vasconcelos e Calado, 2011).

As Diretrizes Curriculares Nacionais do Curso de Graduação em Nutrição (Brasil, 2001) conferem ao nutricionista a função de atuar na promoção, manutenção e recuperação da saúde e na prevenção de doenças de indivíduos ou grupos. A formação do profissional de Nutrição objetiva capacitá-lo para atuar

visando à segurança alimentar e à atenção dietética, em todas as áreas do conhecimento em que alimentação e nutrição se apresentem fundamentais para a promoção, manutenção e recuperação da saúde e para a prevenção de doenças de indivíduos ou grupos populacionais, contribuindo para melhoria da qualidade de vida, pautado em princípios éticos, com reflexão sobre a realidade econômica, política, social e cultural (Brasil, 2001).

Partindo da premissa de que percepções positivas relativas à qualidade de vida podem favorecer a disposição para o aprendizado dos acadêmicos, considerou-se pertinente conhecer a qualidade de vida de futuros promotores de saúde, que lidarão com a vida e a dignidade de outras pessoas, muitas em estado de vulnerabilidade.

\section{Método}

A pesquisa7 foi desenvolvida na Faculdade de Ciências da Saúde da Universidade de Brasília (UnB), com estudantes do curso de Nutrição, no período de agosto de 2010 a agosto de 2011. De acordo com dados do Sistema de Informações Acadêmicas de Graduação, no primeiro semestre de 2010 estavam 
matriculados no curso 224 estudantes, entre eles 190 mulheres e 34 homens. Com base nesses dados, 95\% de confiança, erro padrão máximo igual a 5\%, foi obtida uma amostra aleatória e estratificada por sexo e semestre do curso. A amostra deveria conter quarenta estudantes, com 32 mulheres e oito homens. Contudo, durante a coleta de dados foi detectado que a partir do quarto semestre não havia estudantes do sexo masculino no curso, em decorrência de desistências ou trancamentos de matrícula. Assim, com o objetivo de manter um número representativo de acadêmicos em cada semestre do curso, entrevistaram-se quarenta estudantes - 37 mulheres e três homens.

Tratou-se de um estudo observacional de corte transversal, no qual foram realizadas entrevistas com a aplicação de dois instrumentos de pesquisa: um questionário para identificar os aspectos sociodemográficos dos sujeitos e o World Health Organization Quality of Life Instrument Bref (WHOQOL-BREF), que se reporta aos últimos 15 dias vividos pelos respondentes.

O WHOQOL-BREF, utilizado para avaliar a qualidade de vida de populações adultas, consta de 26 questões. Duas perguntas são gerais e fazem referência à percepção da qualidade de vida e à satisfação com a saúde. As demais representam as 24 facetas que compõem o instrumento original e estão distribuídas em quatro domínios: físico, psicológico, relações sociais e meio ambiente (Fleck et al., 2000). Os domínios e suas respectivas facetas apresentam aspectos objetivos e subjetivos para a avaliação, e as respostas são dadas em uma escala do tipo Likert. As respostas da escala variam de intensidade (nada-extremamente), capacidade (nada-completamente), frequência (nunca-sempre) e avaliação (muito insatisfeito-muito satisfeito e muito ruim-muito bom) (Fleck et al., 1999). O WHOQOL-BREF já foi empregado no Brasil em estudos com idosos (Pereira et al., 2006), pessoas com lesão medular (Bampi, Guilhem e Lima, 2008), com trauma cranioencefálico (Silva et al., 2009), com depressão (Fleck et al., 2002) e portadoras de HIV/Aids (Santos, França Júnior e Lopes, 2007). Também foi utilizado em diversas pesquisas com estudantes universitários (Saupe et al., 2004; Kawakame e Miyadahira, 2005; Catunda e Ruiz, 2008; Hassed et al., 2009; Ramos-Dias et al., 2010; Alves et al., 2010; Leite et al., 2011; Arronqui et al., 2011; Bampi et al., 2013a, 2013b).

A análise dos dados foi efetuada por meio do programa SPSS Statistics 17 e incluiu análises estatísticas descritivas de frequência, tendência central e dispersão, e análise inferencial de comparação entre os domínios. Dos valores encontrados para cada uma das 24 facetas que compõem os domínios, obtiveram-se as medianas das respostas, o que possibilitou verificar quais facetas receberam avaliação positiva ou negativa.

O cálculo dos escores de avaliação da qualidade de vida foi feito separadamente para cada um dos quatro domínios, visto que conceitualmente não está previsto no instrumento um escore global de qualidade de vida. 
O escore transformado 0-100 (ET 0-100) é a escala utilizada na avaliação. Assim, o valor mínimo dos escores de cada domínio é zero e o máximo é 100, de forma que, quanto maior o escore, mais positiva é a avaliação do domínio.

Com a finalidade de comparar os domínios e verificar diferenças estatisticamente significativas, realizou-se o Teste $\mathrm{T}$ de Comparação de Médias para Dados Pareados. Essa avaliação favorece análises comparativas entre os domínios, proporcionando maior visibilidade dos resultados (Saupe et al., 2004).

Efetuou-se análise referente à mediana das respostas em cada uma das questões (facetas) de cada domínio, ou seja, o valor que separa $50 \%$ das respostas, quando estas estão ordenadas. Os valores apontam 1 como a pior resposta e 5 a melhor. Para efeito de uniformização e de modo a possibilitar a comparação, as medianas apresentadas nas facetas relacionadas à dor e ao desconforto, à dependência de tratamentos ou de medicamentos e aos sentimentos negativos foram analisadas de forma invertida, conforme prevê a metodologia da OMS (Fleck et al., 2000).

O projeto foi avaliado e aprovado pelo Comitê de Ética em Pesquisa da Faculdade de Ciências da Saúde da UnB, número de registro CEP 104/09. Esclareceram-se todos os estudantes sobre os objetivos e a metodologia adotados na pesquisa e garantiu-se o sigilo sobre a origem dos dados. A participação voluntária se concretizou por meio da assinatura do termo de consentimento livre e esclarecido. Não há conflitos de interesse.

\section{Resultados}

Os estudantes eram na sua maioria mulheres jovens, com idades entre $18 \mathrm{e}$ 25 anos, idade média 20,6 anos (desvio padrão de 1,81 ano), solteiras, procedentes do Distrito Federal, sem vínculo empregatício.

Com relação às duas questões gerais do WHOQOL-BREF, 82,5\% dos estudantes consideraram sua qualidade de vida boa ou muito boa e $60 \%$ estavam satisfeitos ou muito satisfeitos com suas condições de saúde. Nesta última houve frequência significativa de respostas pouco otimistas, sendo que $22,5 \%$ dos participantes declararam-se nem satisfeitos nem insatisfeitos, e 17,5\% estavam insatisfeitos com sua saúde.

O escore transformado ET 0-100 demonstrou a avaliação média dos quatro domínios. Os escores revelaram que o domínio meio ambiente obteve o mais elevado $(68,36)$, seguido pelos domínios: físico $(66,96)$, relações sociais $(66,87)$ e psicológico $(64,48)$.

Procedendo-se à realização do Teste $\mathrm{T}$ de Comparação de Médias para Dados Pareados, observou-se, entretanto, conforme descrito na Tabela 1, que não existiam diferenças estatisticamente significativas entre as médias dos domínios. 
Tabela 1

Teste T de comparação de médias para dados pareados entre os domínios do WHOQOL-BREF no período de agosto de 2010 a agosto de 2011, Brasília, DF, Brasil, 2013.

\begin{tabular}{lccccc}
\hline Domínio & $\begin{array}{c}\text { Diferença } \\
\text { de médias }\end{array}$ & Erro padrão & Limite inferior & $\begin{array}{c}\text { Limite } \\
\text { superior }\end{array}$ & P-valor \\
\hline Físico & & & & & \\
$\quad$ Psicológico & 2,485 & 2,054 & $-1,669$ & 6,639 & 0,234 \\
$\quad$ Relações sociais & 0,089 & 3,088 & $-6,156$ & 6,335 & 0,977 \\
$\quad$ Meio ambiente & $-1,395$ & 2,742 & $-6,942$ & 4,151 & 0,614 \\
Psicológico & & & & & \\
$\quad$ Físico & $-2,485$ & 2,054 & $-6,639$ & 1,669 & 0,234 \\
$\quad$ Relações sociais & $-2,396$ & 2,442 & $-7,335$ & 2,543 & 0,333 \\
$\quad$ Meio ambiente & $-3,880$ & 2,914 & $-9,774$ & 2,013 & 0,191 \\
Relações sociais & & & & & \\
$\quad$ Físico & 0,595 & 3,088 & $-6,335$ & 6,156 & 0,977 \\
Psicológico & 2,851 & 2,442 & $-2,543$ & 7,335 & 0,333 \\
Meio ambiente & $-1,508$ & 2,974 & $-7,499$ & 4,530 & 0,620 \\
Meio ambiente & & & & & \\
$\quad$ Físico & 1,395 & 2,742 & $-4,151$ & 6,942 & 0,614 \\
Psicológico & 3,880 & 2,914 & $-2,013$ & 9,774 & 0,191 \\
Relações sociais & 1,484 & 2,974 & $-4,530$ & 7,499 & 0,620 \\
\hline
\end{tabular}

Fonte: Os autores.

Com base na análise do box-plot representado pela Figura 1, observa-se que o domínio relações sociais apresenta a maior dispersão em termos de avaliação, tendo, ao mesmo tempo, as piores e as melhores avaliações. $\mathrm{O}$ domínio psicológico apresenta as avaliações mais baixas.

\section{Figura 1}

Escores de avaliação dos domínios que compõem o WHOQOL-BREF no período de agosto de 2010 a agosto de 2011, Brasília, DF, Brasil, 2013.

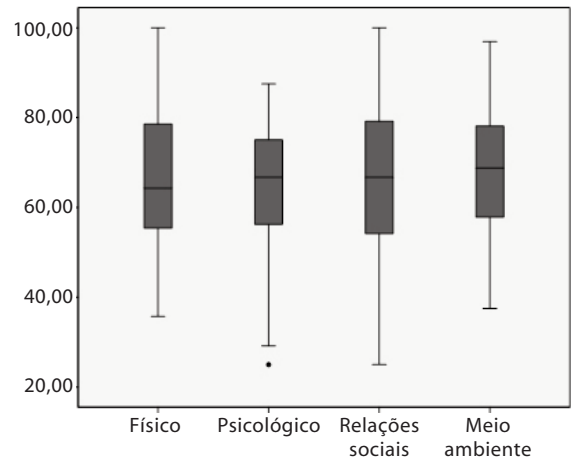

Fonte: Os autores 
O domínio psicológico (Figura 2) é composto por seis facetas e revelou que as facetas sentimentos positivos, crenças pessoais, aceitação da aparência física e autoestima apresentaram mediana 4. A questão sobre concentração apresentou valor mediano de 3. Destaca-se a presença de sentimentos negativos (mau humor, desespero, ansiedade e depressão) em 87,5\% dos estudantes avaliados: 7,5\% apresentaram esses sentimentos algumas vezes, $25 \%$ frequentemente, $50 \%$ muito frequentemente e $5 \%$ sempre.

Figura 2

Mediana das avaliações dos entrevistados nas facetas do domínio psicológico do WHOQOL-BREF no período de agosto de 2010 a agosto de 2011, Brasília, DF, Brasil, 2013.

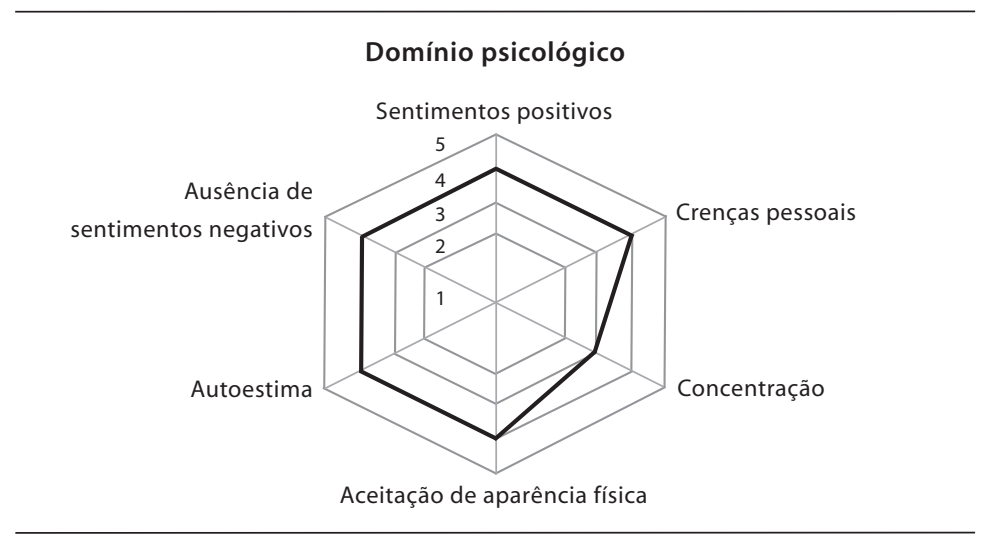

Fonte: Os autores.

Quanto ao domínio relações sociais (Figura 3), os três itens avaliados apresentaram mediana igual a 4, indicando que pelo menos $50 \%$ dos estudantes estão satisfeitos no que diz respeito às suas relações pessoais, ao apoio social e à vida sexual.

\section{Figura 3}

Mediana das avaliações dos entrevistados nas facetas do domínio relações sociais do WHOQOL-BREF no período de agosto de 2010 a agosto de 2011, Brasília, DF, Brasil, 2013.

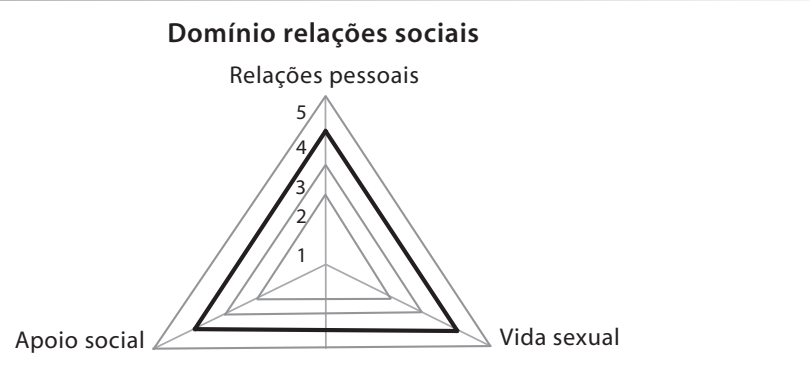

Fonte: Os autores 
No domínio físico (Figura 4), composto de sete questões, as facetas capacidade para o trabalho, locomoção, disposição diante da dor e não dependência de tratamentos tiveram avaliação satisfatória (mediana 4 ou 5). A capacidade de desempenhar as atividades diárias teve avaliação intermediária (mediana 3,5). No entanto, a maioria dos entrevistados $(57,5 \%)$ apresentou insatisfação em relação ao sono e ao grau de energia para o dia a dia, ambos avaliados com mediana 2.

Figura 4

Mediana das avaliações dos entrevistados nas facetas do domínio físico do WHOQOL-BREF no período de agosto de 2010 a agosto de 2011, Brasília, DF, Brasil, 2013.

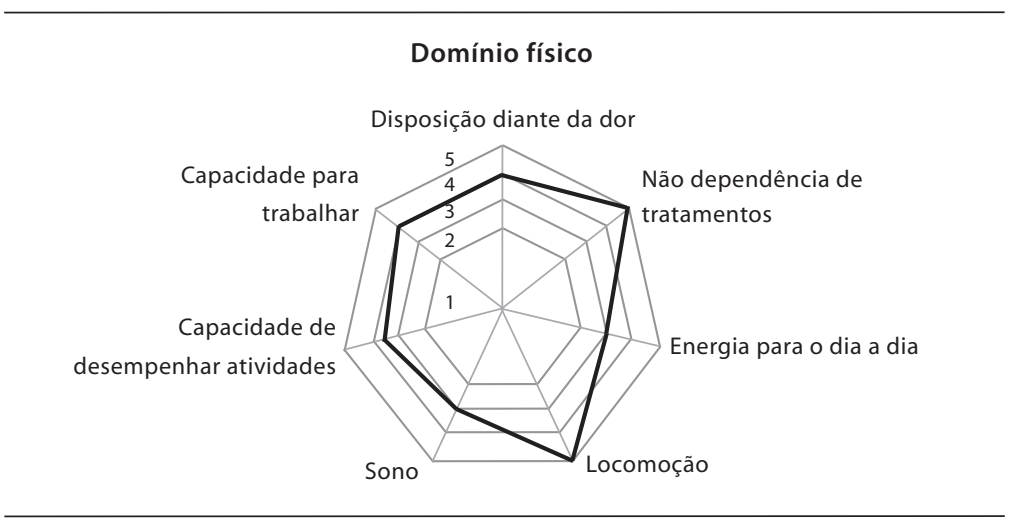

Fonte: Os autores.

O domínio meio ambiente (Figura 5), composto por oito questões, apresentou boas avaliações nas facetas transporte, condições de moradia, segurança, acesso aos serviços de saúde, oportunidades de novas informações - a primeira com mediana 5, a segunda 4,5 e as demais, 4. As questões referentes ao ambiente físico, atividades de lazer e recursos financeiros apontaram mediana 3, demonstrando que 52,7\% dos estudantes avaliaram seu ambiente físico (clima, barulho, poluição, atrativos) como muito pouco ou mais ou menos saudável. Consideráveis $57,9 \%$ dos entrevistados referiram algum grau de comprometimento das oportunidades de lazer, e 57,5\% dos estudantes apresentaram alguma dificuldade em relação aos recursos financeiros para satisfazer suas necessidades $(12,5 \%$ têm muito pouco recurso, $30 \%$ muito recurso, $45 \%$ têm recursos médios e $12,5 \%$ possuem recursos completamente satisfatórios). 
Mediana das avaliações dos entrevistados nas facetas do domínio meio ambiente do WHOQOL-BREF no período de agosto de 2010 a agosto de 2011, Brasília, DF, Brasil, 2013.

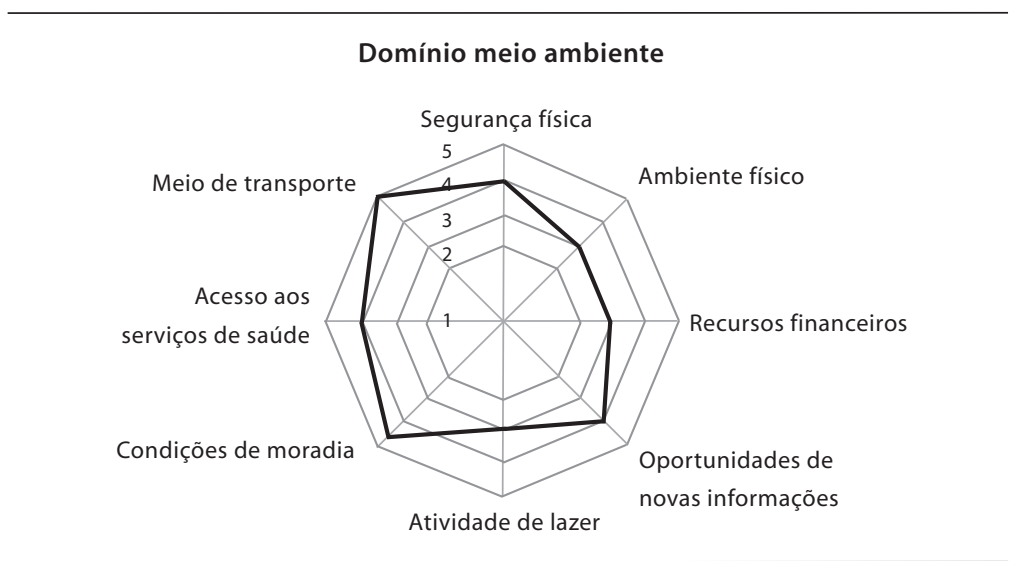

Fonte: Os autores.

\section{Discussão}

No Brasil, os estudos sobre qualidade de vida com estudantes de graduação da área da saúde, especialmente com o uso da metodologia WHOQOL-BREF, vêm tendo foco nas áreas de medicina (Alves et al., 2010; Ramos-Dias et al., 2010; Bampi et al., 2013b) e de enfermagem (Saupe et al., 2004; Kawakame e Miyadahira, 2005; Arronqui et al., 2011; Bampi et al., 2013a). Na área de nutrição foi encontrado somente um artigo (Leite et al., 2011), o que limitou de alguma forma a discussão dos dados encontrados nesta pesquisa.

A maioria dos acadêmicos de Nutrição era do sexo feminino, com idade predominante entre 21 e 25 anos e solteiros, o que corrobora o resultado de estudos similares ao tema e à profissão de nutricionista (Leite et al., 2011). Pesquisas realizadas pelo Instituto Nacional de Estudos e Pesquisas Educacionais Anísio Teixeira (Inep) revelaram que a idade mais frequente dos estudantes que ingressam nas universidades públicas é de 19 anos e a de conclusão do curso é de 23 anos, o que confirma os dados encontrados nesta pesquisa (Brasil, 2009).

A maioria dos acadêmicos declarou que sua qualidade de vida era boa ou muito boa $(82,5 \%)$ e que estava satisfeita ou muito satisfeita com sua saúde $(60 \%)$. Outros estudos conduzidos com acadêmicos de Enfermagem 
(Saupe et al., 2004; Kawakame e Miyadahira, 2005; Arronqui et al., 2011; Bampi et al., 2013a) e de Medicina (Alves et al., 2010; Ramos-Dias et al, 2010; Bampi et al., 2013b), utilizando a mesma metodologia, também encontraram avaliações boas ou muito boas relacionadas à qualidade de vida. Leite e colaboradores (2011) encontraram resultados similares no que se refere à autopercepção da saúde.

Os domínios meio ambiente $(68,3)$, físico $(66,9)$, relações sociais $(66,8)$ e psicológico $(64,4)$ não apresentaram diferenças estatisticamente significativas, ao contrário da pesquisa de Leite e colaboradores (2011), que encontrou diferenças entre todos os domínios, sendo o mais bem avaliado relações sociais $(74,4)$, seguido pelo domínio físico $(71,7)$, psicológico $(67,7)$ e, com o menor escore, o meio ambiente $(67,6)$.

No que diz respeito às facetas que compõem os domínios avaliados, observaram-se algumas especificidades. No domínio psicológico, as facetas sentimentos positivos, autoestima, crenças pessoais e aceitação de aparência física receberam avaliação positiva, similar aos achados dos acadêmicos de Enfermagem utilizando a mesma metodologia (Bampi et al., 2013a). A faceta concentração obteve mediana 3, o que significa que pelo menos $50 \%$ dos entrevistados não estavam satisfeitos nem insatisfeitos com sua capacidade de se concentrar - associando-se com as facetas do domínio físico, sono e energia para o dia a dia, que também obtiveram avaliação mediana, demonstrando algum grau de insatisfação dos acadêmicos em tais quesitos. Nesse domínio, chama a atenção a presença de sentimentos negativos (mau humor, desespero, ansiedade e depressão) em 87,5\% dos estudantes avaliados, resultado semelhante encontrado em acadêmicos de Enfermagem e Medicina (Bampi et al., 2013a, 2013b).

No domínio relações sociais, os itens relações pessoais, apoio social e vida sexual apresentaram mediana 4, demonstrando que os entrevistados estão satisfeitos com esses quesitos. Em estudos similares com universitários, a avaliação nesse domínio foi satisfatória (Saupe et al., 2004; Marconi et al., 2004; Leite et al., 2011; Bampi et al., 2013a, 2013b).

No domínio físico, as facetas locomoção e não dependência de tratamentos apresentaram as melhores avaliações, com pelo menos metade dos entrevistados classificando-as como 5, ou seja, estavam muito satisfeitos. As questões sobre sono e energia para o dia a dia receberam as piores avaliações, mediana 3. Os dados demonstraram que 47,5\% dos entrevistados estavam insatisfeitos ou muito insatisfeitos com o sono e que $70 \%$ avaliaram sua energia para o dia a dia como média ou muito pouca, podendo, portanto, ocasionar déficits de atenção e prejudicar sua condição de aprendizado (Lim e Dinges, 2008; Soares e Almondes, 2012).

No domínio meio ambiente, as facetas transporte (mediana 5) e condições de moradia (mediana 4,5) foram consideradas muito satisfatórias, podendo 
revelar que esses estudantes conseguem se locomover numa cidade cosmopolita com precárias condições de transporte público e possuem condições adequadas de moradia. Vale destacar que a pesquisa ocorreu na fase inicial da implantação da política de reestruturação e expansão das universidades federais, permeada por reformas e ampliações da estrutura física, retratando um descontentamento em termos dessas condições para acadêmicos que realizam curso em período integral. Ainda nesse domínio, a maioria $(57,5 \%)$ tem muito pouco $(17,5 \%)$ ou médias $(40 \%)$ oportunidades de lazer. A insatisfação dos futuros nutricionistas com a falta de tempo para o lazer pode relacionar-se com a carga horária exigida para o curso e as exigências curriculares, uma vez que a totalidade dos entrevistados não possui vínculo empregatício e dedica-se integralmente ao curso.

Embora a metodologia de avaliação WHOQOL-BREF não contemple a dimensão político-institucional em que os sujeitos estão inseridos, é importante refletir no sentido de que a transição ainda vivenciada pela reforma estabelecida pelas Diretrizes Curriculares Nacionais dos Cursos de Graduação em Enfermagem, Medicina e Nutrição (Brasil, 2001) pode ter influenciado a avaliação dos participantes desta pesquisa - o que se demonstrou nos domínios físico (energia para o dia a dia, sono e capacidade para desempenhar atividades diárias), psicológico (concentração, presença de sentimentos negativos) e meio ambiente (ambiente físico, recursos financeiros e oportunidade de atividades de lazer), que têm estreita correlação com o desempenho de aprendizado desses estudantes. Nesse sentido, embora a reforma do ensino superior brasileiro fundamente-se em diretrizes que ofereçam um percurso humanizado e mais bem contextualizado, de forma que os profissionais sejam capazes de atuar com autonomia e discernimento, assegurando-se a qualidade e a humanização na prestação do atendimento e a integralidade das ações, na vida do sujeito no decorrer de sua formação e dos estudos pregressos, tal proposta parece dissonante para esses jovens. Os resultados encontrados nesta pesquisa e em diversas da mesma natureza com estudantes oriundos de universidades públicas demonstram que fatores ligados à construção da vida acadêmica são avaliados negativamente (Bampi et al., 2013a, 2013b; Alves et al., 2010). Assim, a formação de profissionais de saúde generalistas, críticos e reflexivos parece estar tolhendo aspectos fundamentais para a sua qualidade de vida.

É importante lembrar que algumas pesquisas (Oliveira e Ciampone, 2006; Eurich e Kluthcovsky, 2008) com estudantes de Enfermagem apontam ser necessário direcionar maior atenção aos aspectos subjetivos da graduação, uma vez que o aluno deve aprender, além da técnica e da lógica, a lidar com eventos que farão parte de sua rotina profissional. Tais estudos destacam a falta de apoio aos estudantes por parte das estruturas acadêmicas e dos docentes, revelando que quando há algum apoio este é prestado 
prioritariamente na esfera pedagógica, sendo os aspectos psicológico e emocional postergados.

Ainda que certos prejuízos na qualidade de vida possam não estar diretamente relacionados à graduação, é preciso pensar em um suporte mais eficaz aos estudantes como forma de apoiar o enfrentamento das situações que eles experimentarão durante o curso, sejam relativas ao contexto acadêmico, sejam ao seu contexto individual. Eficaz, inclusive, na forma de sensibilizá-los quanto à importância dessa condição em suas vidas pessoais e profissionais, pois estarão envolvidos com o cuidado de outras pessoas e com a promoção da qualidade de vida de coletividades.

Outro aspecto que pode influenciar a percepção dos estudantes é a própria escolha da profissão. Em estudo similar e comparado entre estudantes da área de Nutrição e de Psicologia, a qualidade de vida e a condição de saúde foram avaliadas mais satisfatoriamente pelos estudantes de $\mathrm{Nu}$ trição. Os autores sugerem que a preocupação central do nutricionista direciona-se ao prazer que envolve a vida do ser humano, enquanto o psicólogo direciona sua atenção ao sofrimento (Marconi et al., 2004). No entanto, é preciso reconhecer que a nutrição não se resume ao prazer de comer, ao passo que a psicologia não se limita à angústia psíquica. De fato, embora isso possa ser considerado uma verdade para alguns profissionais já inseridos no mercado de trabalho e com atribuições bastante específicas, talvez não se aplique ao estudante, que está em contato com as mais diversas áreas de atuação e nem sempre apresenta a vivência prática de sua futura profissão.

Apesar de o reconhecimento dessa profissão ter ocorrido em 1962, recentemente tem se verificado uma diversificação e ampliação da atuação do nutricionista: nutrição clínica, alimentação coletiva, saúde coletiva, ensino (docência), nutrição esportiva, indústria de alimentos e marketing em alimentação e nutrição (Vasconcelos e Calado, 2011). Tal diversificação de possibilidades ao graduando pode facilitar melhor identificação com a profissão, tornando-a mais satisfatória em termos de formação e aprendizado.

As pesquisas sobre esse tema convidam à reflexão sobre como a qualidade de vida é afetada durante a formação profissional. Além da graduação baseada no currículo, é fundamental o aprimoramento de estratégias que proporcionem qualidade de vida ao ser humano que se pretende formar.

\section{Conclusão}

O estudo permitiu conhecer a percepção sobre qualidade de vida dos estudantes de Nutrição da UnB, assim como os fatores que influenciaram essa perspectiva teórico-metodológica de avaliação. 
A qualidade de vida foi mensurada positivamente por $82,5 \%$ dos respondentes. Não obstante, é importante refletir que se trata de uma população jovem e em franco processo de formação profissional que pode estar sinalizando fragilidades em termos da sua condição de vida física e emocional, uma vez que mais da metade deles relataram a presença de sentimentos negativos (mau humor, desespero, ansiedade e depressão) e a insatisfação (insatisfeitos ou muito insatisfeitos) com as facetas sono, energia para o dia a dia, capacidade de concentração e oportunidade de lazer. Esses aspectos podem impactar no rendimento escolar ou mesmo se inter-relacionar em termos de uma qualidade de vida desejável.

Em vista dos resultados encontrados e corroborando outros estudos sobre o mesmo tema, é importante repensar a articulação de políticas públicas, especialmente as direcionadas à saúde e à educação, que possam compreender a abrangência do tema. Prover apoio pedagógico, social e psicológico aos acadêmicos de modo integrado ao contexto organizacional em que estão inseridos faz-se relevante num contexto de inclusão social e multidiversidade cultural cada vez mais presente nas universidades públicas brasileiras.

\section{Colaboradores}

A coleta de dados foi realizada por Mariana Fernandes Pereira. Ana Carolina Oliveira Campos fez a análise estatística. A elaboração, a análise, a discussão e a revisão do artigo foram feitas por Solange Baraldi e Luciana Neves da Silva Bampi. Aline Brandão Mariath colaborou na discussão e na revisão final do artigo, enquanto Dirce Bellezi Guilhem contribuiu na revisão. 
Resumen Este estudio tuvo como objetivo evaluar la calidad de vida de los estudiantes de Nutrición, con el fin de sustentar reflexiones sobre el proceso de formación en salud. Se trató de un estudio observacional de corte transversal, realizado con cuarenta estudiantes distribuidos en todos los semestres del curso durante el período de agosto de 2010 a agosto de 2011 . La recolección de datos se realizó mediante un cuestionario específico -aspectos sociodemográficos- y del instrumento World Health Organization Quality of Life Instrument Bref. Se realizaron análisis estadísticos descriptivos de frecuencia, tendencia central y dispersión y análisis inferencial de comparación entre los dominios. Estos presentaron las siguientes puntuaciones: medio ambiente $(68,17)$, relaciones sociales $(66,67)$, física $(66,07)$ y psicológica $(63,82)$. Las facetas sueño, energía para el día a día, capacidad de concentración, oportunidades de actividades recreativas, recursos financieros, ambiente físico y sentimientos negativos afectaron negativamente la calidad de vida de los encuestados e interfirieron en el rendimiento académico. Estos hallazgos corroboran otros estudios sobre estudiantes de pregrado, que revelan que la calidad de vida de estos jóvenes exige la mejora de las estructuras físicas y ambientales, del apoyo pedagógico, social y psicológico en un contexto de inclusión social y de multidiversidad cultural cada vez más presente en las universidades públicas brasileñas.

Palabras clave calidad de vida; percepción; estudiantes; pregrado; Nutrición.

\section{Notas}

1 Universidade de Brasília, Faculdade de Ciências da Saúde, Departamento de Enfermagem, Brasília, Distrito Federal, Brasil.

Doutora em Enfermagem pela Universidade de São Paulo.

<solbaraldi@unb.br>

Correspondência: SQN, 205, H, apartamento 302, CEP 70843-080, Brasília, Distrito Federal, Brasil.

2 Universidade de Brasília, Faculdade de Ciências da Saúde, Departamento de Enfermagem, Brasília, Distrito Federal, Brasil.

Doutora em Ciências da Saúde pela Universidade de Brasília.

<lbampi@unb.br>

3 Secretaria de Estado de Saúde do Distrito Federal, Hospital Regional de Santa Maria, Santa Maria, Brasília, Distrito Federal, Brasil.

Graduada em Enfermagem pela Universidade de Brasília, Brasil.

$<$ marifp88@gmail.com>

4 Universidade de Brasília, Faculdade de Ciências da Saúde, Departamento de Enfermagem, Brasília, Distrito Federal, Brasil.

Doutora em Ciências da Saúde pela Universidade de Brasília.

<guilhem@unb.br>

5 Câmara dos Deputados, Departamento Médico, Brasília, Distrito Federal, Brasil.

Mestre pelo Programa de Nutrição em Saúde Pública da Faculdade da Saúde Pública da Universidade de São Paulo.

<alinemariath@camara.leg.br> 
6 Universidade Cruzeiro do Sul, Brasília, Distrito Federal, Brasil.

Pós-graduada em Econometria e Finanças Quantitativas pela Fundação Universa.

<carol.estat@gmail.com>

7 A pesquisa "Qualidade de Vida em Estudantes de Graduação das Faculdades de Ciências da Saúde e de Medicina da Universidade de Brasília", que deu origem a este artigo, foi fruto do trabalho do Grupo do Laboratório de Bioética e Ética em Pesquisa da Universidade de Brasília no bojo do projeto Qualidade de Vida em Estudantes de Graduação, idealizado e coordenado por Luciana das Neves da Silva Bampi, em cooperação com Solange Baraldi e Dirce Guilhem.

\section{Referências}

ALMEIDA FILHO, Naomar. O conceito de saúde: ponto cego da epidemiologia? Revista Brasileira de Epidemiologia, São Paulo, v. 13, n. 1, p. 4-20, 2000.

ALVES, João G. B. et al. Qualidade de vida em estudantes de medicina no início e final do curso: avaliação pelo WHOQOL-BREF. Revista Brasileira de Educação Médica, Rio de Janeiro, v. 34, n. 1, p. 91-96, mar. 2010.

ARRONQUI, Grazielle V. et al. Percepção de graduandos de Enfermagem sobre sua qualidade de vida. Acta Paulista de Enfermagem, São Paulo, v. 24, n. 6, p. 762-765, nov./dez. 2011.

BAMPI, Luciana N. S.; GUILHEM, Dirce; LIMA, David Duarte. Qualidade de vida em pessoas com lesão medular traumática: um estudo com o WHOQOL-BREF. Revista Brasileira de Epidemiologia, São Paulo, v. 11, n. 1, p. 67-77, mar. 2008.

BAMPI, Luciana N. S. et al. Percepção sobre qualidade de vida de estudantes de graduação em Enfermagem. Revista Gaúcha de Enfermagem, Porto Alegre, v. 34, n. 2, p. 125-132, 2013a.

BAMPI, Luciana N. S. et al. Qualidade de vida de estudantes de Medicina da Universidade de Brasília. Revista Brasileira de Educação Médica, Rio de Janeiro, v. 37, n. 2, p. 217-225, 2013b.
BRASIL. Constituição da República Federativa do Brasil de 1988. 1988. Disponível em: $<$ www.planalto.gov.br/ccivil_03/constituicao/ constituicaocompilado.htm $>$. Acesso em: maio 2010.

BRASIL. Ministério da Educação. Diretrizes Curriculares Nacionais para Cursos de Graduação em Enfermagem, Medicina e Nutrição. 2001. Disponível em: <http://portal.mec.gov. br/dmdocuments/ces1133.pdf $>$. Acesso em: jul. 2012.

BRASIL. Ministério da Educação. Instituto Nacional de Estudos e Pesquisas Educacionais Anísio Teixeira. Resumo Técnico: Censo da Educação Superior de 2009. Disponível em: <http://download.inep.gov.br/download/ superior/censo/2009/resumo_tecnico_2009.pdf>.

CATUNDA, Maria A. P.; RUIZ, Valdete M. Qualidade de vida de universitários. Pensamento Plural, Pelotas, v. 2, n. 1, p. 22-31, 2008.

CECCIM, Ricardo B.; FEUERWERKER, Laura M. Mudança na graduação das profissões de saúde sob o eixo da integralidade. Cadernos de Saúde Pública, Rio de Janeiro, v. 20, n. 5, p. 1.400-1.410, set./out. 2004.

EURICH, Rosane B.; KLUTHCOVSKY, Ana Cláudia G. C. Avaliação da qualidade de vida de acadêmicos de graduação em Enfermagem 
do primeiro e quarto anos: influência das variáveis sociodemográficas. Revista de Psiquiatria do Rio Grande do Sul, Porto Alegre, v. 30, n. 3, p. 211-220, dez. 2008.

FLECK, Marcelo P. A. et al. Desenvolvimento da versão em português do instrumento de avaliação de qualidade de vida da OMS (WHOQOL-100). Revista Brasileira de Psiquiatria, São Paulo, v. 21, n. 1, p. 19-28, mar. 1999.

FLECK, Marcelo P. A. et al. Aplicação da versão em português do instrumento abreviado de avaliação da qualidade de vida 'WHOQOL-BREF'. Revista de Saúde Pública, São Paulo, v. 34, n. 2, p. 178-183, abr. 2000.

FLECK, Marcelo P. A. et al. Associação entre sintomas depressivos e funcionamento social em cuidados primários de saúde. Revista de Saúde Pública, São Paulo, v. 36, n. 4, p. 431-438, ago. 2002.

HASSED, Craig et al. Enhancing the health of medical students: outcomes of an integrated mindfulness and lifestyle program. Advances in Health Sciences Education, Boston, v. 14, n. 3, p. 387-398, ago. 2009.

KAWAKAME, Patrícia M. G.; MIYADAHIRA, Ana Maria Kazue. Qualidade de vida de estudantes de graduação em Enfermagem. Revista da Escola de Enfermagem da USP, São Paulo, v. 39, n. 2, p. 164-172, jun. 2005.

LEITE, Ana C. B. et al. Qualidade de vida e condições de saúde de acadêmicos de $\mathrm{Nu}$ trição. Revista Espaço para a Saúde, Londrina, v. 13, p. 82-90, dez. 2011.

LIM, Julian; DINGES, David F. Sleep deprivation and vigilant attention. Annals of the New York Academy of Sciences, New York, v. 1.129 , p. 305-322, maio 2008.

MARCONI, Eda Custódio et al. Qualidade de vida entre universitárias: estudos preliminares com o WHOQOL-BREF. Boletim
Academia Paulista de Psicologia, São Paulo, v. 24, n. 3, p. 47-57, set./dez. 2004.

OLIVEIRA, Raquel A.; CIAMPONE, Maria H. T. A universidade como espaço promotor de qualidade de vida: vivências e expressões dos alunos de Enfermagem. Texto e Contexto Enfermagem, Florianópolis, v. 15, n. 2, p. 254-261, abr./jun. 2006.

ORGANIZAÇÃO MUNDIAL DA SAÚDE(OMS). Constituição da Organização Mundial da Saúde (OMS/WHO), Nações Unidas, 1946. Biblioteca Virtual de Direitos Humanos. Disponível em: <www.direitoshumanos.usp. br/index.php/OMS-Organiza \% C3\% A 7\% C3\% A 3o-Mundial-da-Sa \% C3\%BAde/ constituicao-da-organizacao-mundial-dasaude-omswho.html>. Acesso em: jun. 2012.

ORGANIZAÇÃO MUNDIAL DA SAÚDE. Divisão de Saúde Mental. Grupo WHOQOL. Grupo de Estudos em Qualidade de Vida. Versão em português dos instrumentos de avaliação de qualidade de vida (WHOQOL). Porto Alegre: Universidade Federal do Rio Grande do Sul, 1998. Disponível em: <www. ufrgs.br/psiquiatria/psiq/whoqol.html>. Acesso em: jun. 2012.

PEREIRA, Renata J. et al. Contribuição dos domínios físico, social, psicológico e ambiental para a qualidade de vida global de idosos. Revista de Psiquiatria do Rio Grande do Sul, Porto Alegre, v. 28, n. 1, p. 27-38, abr. 2006.

RAMOS-DIAS, João C. et al. Qualidade de vida em cem alunos do curso de Medicina de Sorocaba - PUC/SP. Revista Brasileira de Educação Médica, Rio de Janeiro, v. 34, n. 1, p. 116-123, mar. 2010.

SANTOS, Elisabete C. M.; FRANÇA JÚNIOR, Ivan; LOPES, Fernanda. Qualidade de vida de pessoas vivendo com HIV/Aids em São Paulo. Revista de Saúde Pública, São Paulo, v. 41, s. 2, p. 64-71, dez. 2007.

SAUPE, Rosita et al. Qualidade de vida dos acadêmicos de Enfermagem. Revista Latino- 
-Americana de Enfermagem, Ribeirão Preto, SP, v. 12, n. 4, p. 636-642, jul./ago. 2004.

SILVA, Cleuza B. et al. Avaliação da qualidade de vida de pacientes com trauma cranioencefálico. Fisioterapia e Pesquisa, São Paulo, v. 16, n. 4, p. 311-315, dez. 2009.

SOARES, Cibele S.; ALMONDES, Katie M. Sono e cognição: implicações da privação do sono para a percepção visual e visuoespacial. Psico, Porto Alegre, v. 43, n. 1, p. 85-92, jan./mar. 2012.
VASCONCELOS, Assis G.; CALADO, Carmem L. A. Profissão nutricionista: 70 anos de história no Brasil. Revista de Nutrição, Campinas, v. 24, n. 4, p. 605-617, jul./ago. 2011.

Recebido em 18/10/2012

Aprovado em 11/06/2014 
